\title{
Thrombin-Binding Aptamer Quadruplex Formation: AFM and Voltammetric Characterization
}

\author{
Victor Constantin Diculescu, ${ }^{1}$ Ana-Maria Chiorcea-Paquim, ${ }^{1}$ Ramon Eritja, ${ }^{2}$ \\ and Ana Maria Oliveira-Brett ${ }^{1}$
}

${ }^{1}$ Departamento de Quimica, Faculdade de Ciências e Tecnologia, Universidade de Coimbra, 3004-535 Coimbra, Portugal

${ }^{2}$ Institute for Research in Biomedicine, IQAC-CSIC, CIBER-BBN Networking Centre on Bioengineering, Biomaterials and Nanomedicine, 08028 Barcelona, Spain

Correspondence should be addressed to Ana Maria Oliveira-Brett, brett@ci.uc.pt

Received 15 January 2010; Accepted 29 March 2010

Academic Editor: Lea Spindler

Copyright (c) 2010 Victor Constantin Diculescu et al. This is an open access article distributed under the Creative Commons Attribution License, which permits unrestricted use, distribution, and reproduction in any medium, provided the original work is properly cited.

\begin{abstract}
The adsorption and the redox behaviour of thrombin-binding aptamer (TBA) and extended TBA (eTBA) were studied using atomic force microscopy and voltammetry at highly oriented pyrolytic graphite and glassy carbon. The different adsorption patterns and degree of surface coverage were correlated with the sequence base composition, presence/absence of $\mathrm{K}^{+}$, and voltammetric behaviour of TBA and eTBA. In the presence of $\mathrm{K}^{+}$, only a few single-stranded sequences present adsorption, while the majority of the molecules forms stable and rigid quadruplexes with no adsorption. Both TBA and eTBA are oxidized and the only anodic peak corresponds to guanine oxidation. Upon addition of $\mathrm{K}^{+}$ions, TBA and eTBA fold into a quadruplex, causing the decrease of guanine oxidation peak and occurrence of a new peak at a higher potential due to the oxidation of G-quartets. The higher oxidation potential of G-quartets is due to the greater difficulty of electron transfer from the inside of the quadruplex to the electrode surface than electron transfer from the more flexible single strands.
\end{abstract}

\section{Introduction}

Since it has been recognized that G-rich nucleic acid sequences can adopt intermolecular and intramolecular quadruplex structures stabilized by G-quartets [1], Scheme 1, there is substantial interest in the role of quadruplex formation, largely as a consequence of the identification of G-quadruplex-forming sequences in the genome (e.g., telomers, several oncogenes and aptamers) [2-4]. Despite the intensive studies on the role of G-quartet formation in vivo, there is considerable interest in the therapeutic potential of quadruplex oligodeoxynucleotides (ODNs).

Aptamers are nucleic acid sequences (DNA or RNA) selected in vitro from large combinatorial pools to bind to specific targets [5-7]. Aptamers exhibit a strong and specific binding affinity towards their targets and can be simply synthesized via cost-effective and readily automated routes $[8,9]$. More important, the structure and conformation of aptamers can be rationally designed and tailor-made to be selectively responsive to various kinds of targets ranging from small molecules [9] to biological molecules $[7,10,11]$ and even cells $[12,13]$. Aptamers possess significant advantages over other recognition molecules, such as antibodies, due to their small size, chemical simplicity, and flexibility.

One of the most preeminent examples of the in vitro selection of DNA oligonucleotides for targeting a specific protein is the thrombin-binding aptamer (TBA), Scheme 2. Thrombin is a serine protease and a coagulation protein in the blood stream that has many effects in the coagulation mechanism. Activation of thrombin is crucial in physiological and pathological coagulation. Various rare diseases involving thrombin have been described. Blood from a ruptured cerebral aneurysm clots around a cerebral artery and releases thrombin, which can induce an acute and prolonged narrowing of the blood vessel, potentially resulting in cerebral ischemia and infarction (stroke). Because of its importance in anticlotting therapeutics, TBA has 


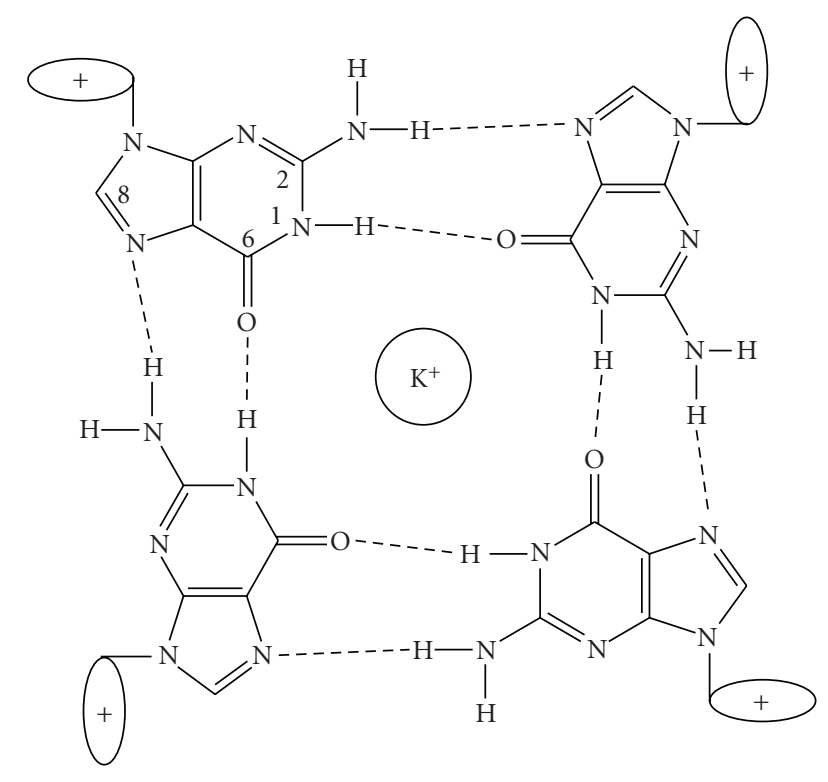

Scheme 1: Schematic representation of the G-quartet.

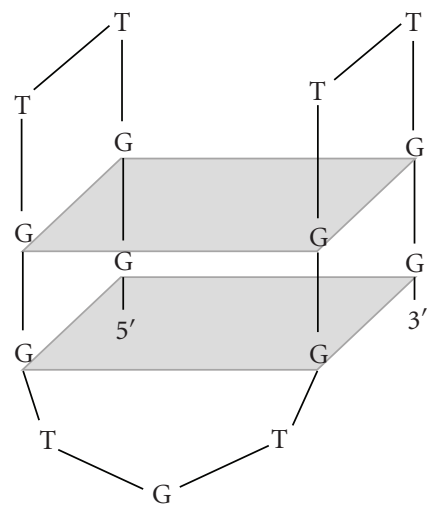

(a)

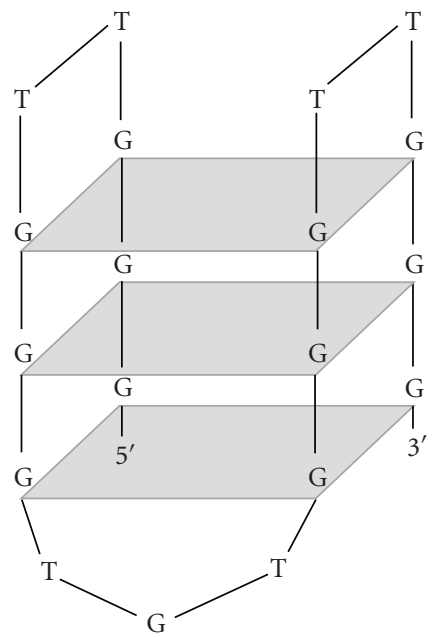

(b)

Scheme 2: Schematic representation of the antiparallel G-quadruplex structures of (a) TBA and (b) eTBA.

been studied extensively. It has been shown that in the presence of alkali metals $[4,7,14]$, TBA forms an antiparallel intramolecular quadruplex consisting of two G-quartets connected by two TT loops and one TGT loop. TBA has also been used for development of electrochemical biosensors for the detection of thrombin [10]. However, no publication has shown the adsorption and voltammetric behaviour of these G-quartet forming ODNs.

The present paper is the first report of a voltammetric and atomic force microscopy (AFM) study of two different thrombin-binding aptamer sequences. The process of adsorption of TBA (5'-GGTTGGTGTGGTTGG-3') and extended TBA (eTBA, $5^{\prime}$-GGGTTGGGTGTGGGTTGGG-3' was studied on a highly oriented pyrolytic graphite (HOPG) electrode using acoustic AC mode AFM (AAC Mode AFM). The electrochemical oxidation of these TBA sequences has been also performed by differential pulse (DP) voltammetry using a glassy carbon (GC) electrode.

\section{Experimental}

2.1. Materials and Reagents. The ODN sequences TBA (5'-GGTTGGTGTGGTTGG-3') and eTBA (5'-GGGTTGGGTGTGGGTTGGG-3'), Scheme 2, were synthesized on an ABI 3400 DNA Synthesizer (Applied Biosystems, Foster City, CA, USA) using the 200-nmol scale synthesis cycle [7]. All 
solutions were prepared using analytical grade reagents and purified water from a Millipore Milli-Q system (conductivity $\leq 0.1 \mu \mathrm{S} \mathrm{cm}^{-1}$ ). Stock TBA and eTBA solutions were prepared in Milly Q water and kept at $4^{\circ} \mathrm{C}$. Before each experiment, solutions of either TBA or eTBA were freshly prepared by dilution of the appropriate quantity in $\mathrm{pH} 7.0 \quad 0.1 \mathrm{M}$ phosphate buffer $\left(0.2 \mathrm{M} \mathrm{Na}_{2} \mathrm{HPO}_{4}+0.2 \mathrm{M} \mathrm{NaH}_{2} \mathrm{PO}_{4}\right)$.

Microvolumes were measured using EP-10 and EP-100 Plus Motorized Microliter Pippettes (Rainin Instrument Co. Inc., Woburn, USA). The $\mathrm{pH}$ measurements were carried out with a Crison micropH $2001 \mathrm{pH}$-meter with an Ingold combined glass electrode. All experiments were done at room temperature $\left(25 \pm 1^{\circ} \mathrm{C}\right)$.

2.2. Atomic Force Microscopy. HOPG, grade ZYB of dimensions $15 \times 15 \times 2 \mathrm{~mm}^{3}$, from Advanced Ceramics Co., USA, was used as a substrate in the AFM study. The HOPG was freshly cleaved with adhesive tape prior to each experiment and imaged by AFM in order to establish its cleanliness.

AFM was performed in the AAC mode AFM, with a PicoScan controller from Agilent Technologies, Tempe, AZ, USA. All the AFM experiments were performed with a CS AFM S scanner with a scan range of $6 \mu \mathrm{m}$ in $x-y$ and $2 \mu \mathrm{m}$ in $z$, from Agilent Technologies. AppNano type FORT of $225 \mu \mathrm{m}$ length, $3.0 \mathrm{~N} \mathrm{~m}^{-1}$ spring constants, and $47-76 \mathrm{kHz}$ resonant frequencies in air (Applied NanoStructures, Inc., USA) were used. All AFM images were topographical and were taken with 256 samples/line $\times 256$ lines and scan rates of 0.8-2.0 lines s ${ }^{-1}$. When necessary, the AFM images were processed by flattening in order to remove the background slope and the contrast and brightness were adjusted.

2.3. Sample Preparation for AFM. The TBA and eTBA modified HOPG surfaces were obtained by spontaneous adsorption, by depositing $200 \mu \mathrm{L}$ samples of $1 \mu \mathrm{g} \mathrm{mL}^{-1} \mathrm{TBA}$ or eTBA solutions in $\mathrm{pH}$ 7.0 0.1 M phosphate buffer onto the freshly cleaved HOPG surface, during 3 minutes. The excess of solution was gently cleaned with a jet of Millipore MilliQ water, and the HOPG with adsorbed ODN molecules was then dried in a sterile atmosphere and imaged by AAC Mode AFM in air.

2.4. Voltammetric Parameters and Electrochemical Cells. Voltammetric experiments were carried out using a $\mu$ Autolab running with GPES 4.9 software, Eco-Chemie, The Netherlands. Measurements were carried out using a $\mathrm{GC}(d=1.5 \mathrm{~mm})$ working electrode, a Pt wire counter electrode, and a $\mathrm{Ag} / \mathrm{AgCl}$ as reference, in a $0.25 \mathrm{~mL}$ electrochemical cell. The experimental conditions for DP voltammetry were pulse amplitude $50 \mathrm{mV}$, pulse width $70 \mathrm{~ms}$, scan rate $5 \mathrm{mV} \mathrm{s}^{-1}$.

The GC electrode was polished using diamond spray (particle size $1 \mu \mathrm{m}$ ) before each experiment. After polishing, the electrode was rinsed thoroughly with Milli-Q water and placed in supporting electrolyte and various DP voltammograms were recorded until a steady state baseline voltammogram was obtained. This procedure ensured very reproducible experimental results.
2.5. Acquisition and Presentation of Voltammetric Data. The DP voltammograms were baseline-corrected using the moving average with a step window of $2 \mathrm{mV}$ included in GPES version 4.9 software. This mathematical treatment improves the visualization and identification of peaks over the baseline without introducing any artefact, although the peak height is in some cases reduced $(<10 \%)$ relative to that of the untreated curve. Nevertheless, this mathematical treatment of the original voltammograms was used in the presentation of all experimental voltammograms for a better and clearer identification of the peaks. The values for peak current presented in all graphs were determined from the original untreated voltammograms after subtraction of the baseline.

\section{Results and Discussion}

3.1. AFM Characterisation of Spontaneously Adsorbed TBA and eTBA. The capacity of TBA and eTBA molecules to interact and adsorb spontaneously on the HOPG electrode forming different morphological films was investigated by AFM in air. The spontaneous adsorption of the ODN sequences was obtained using concentrations of $1 \mu \mathrm{g} \mathrm{mL}-1$ TBA or eTBA in pH 7.0 0.1 M phosphate buffer as described in Section 2.3. The relationship between the degree of surface coverage with adsorbed molecules, the base composition in the ODN sequence and the presence/absence of $\mathrm{K}^{+}$cations will be discussed.

An atomically flat HOPG electrode was used as a substrate with less than $0.06 \mathrm{~nm}$ of root-mean-square (r.m.s.) roughness for a $1000 \times 1000 \mathrm{~nm}^{2}$ surface area. The GC electrode used for the voltammetric characterisation was much rougher, with $2.10 \mathrm{~nm}$ r.m.s. roughness for the same surface area, therefore unsuitable for AFM surface characterisation. Furthermore, the experiments using GC and HOPG electrodes showed similar electrochemical behaviour.

AFM topographical images in air of the TBA modified HOPG electrode showed that the TBA adsorbs spontaneously onto HOPG, showing the formation of two different adsorbed structures: a thin and incomplete network film, Figure 1(a), and aggregates with spherical and rodlike shapes, marked with white arrows in the images, Figure 1(b). The network was composed of small molecules adsorbed side by side that formed close-packed "polymerlike" chains, with many pores, corresponding to the dark regions in the images, and exposed HOPG surface at the bottom of the pores, Figure 1(a). The measured thickness of the TBA layer was calculated from cross section analysis as being $0.8 \pm 0.1 \mathrm{~nm}$. The average height and standard deviation of the spherical aggregates was $1.9 \pm 0.5 \mathrm{~nm}$, Figure 1(b), higher than the values obtained for the TBA lattices.

Similar results were obtained for the eTBA molecules, which present four extra guanines in the base sequence. The AFM images in air also showed a densely packed $0.8 \pm 0.1 \mathrm{~nm}$ height self-assembled network with looped arms, Figure 2(a), and small TBA fragments of $0.8 \pm 0.1 \mathrm{~nm}$ height with embedded $1.5 \pm 0.2 \mathrm{~nm}$ height aggregates (marked with white arrows), Figure 2(b). 


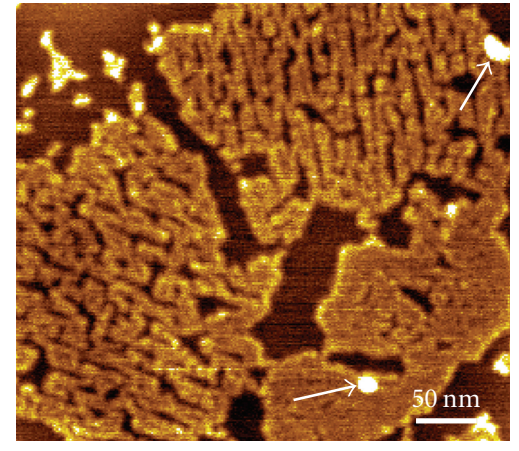

(a)

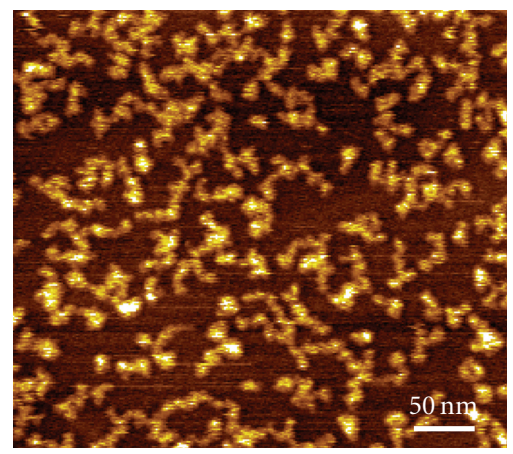

(c)

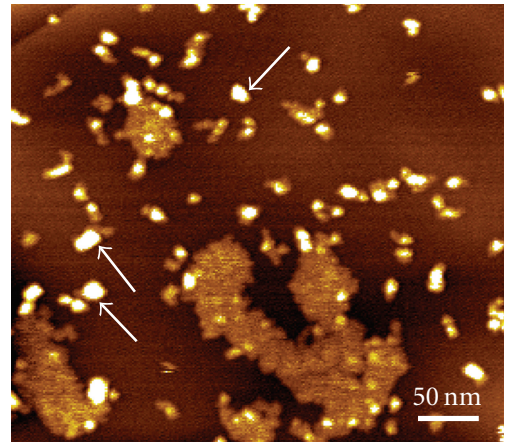

(b)

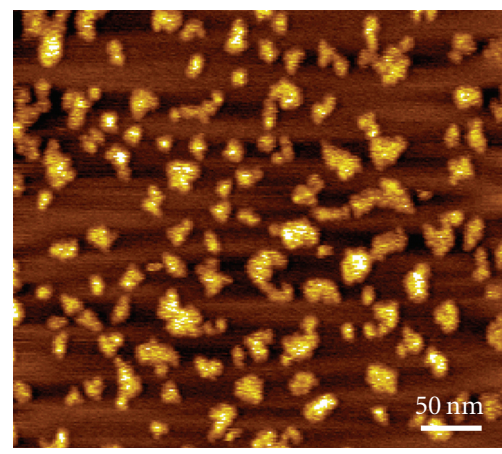

(d)

FIGURE 1: AFM topographical images in air of TBA molecules, immobilized onto HOPG by spontaneous adsorption during 3 minutes, from solutions of $1 \mu \mathrm{g} \mathrm{mL}^{-1}$ TBA in pH 7.0 $0.1 \mathrm{M}$ phosphate buffer: (a, b) in the absence and (c, d) in the presence of $100 \mathrm{mM} \mathrm{K}^{+}$, after (c) 1 hour and (d) 24 hours of incubation.

The adsorption mechanism of the nucleic acid molecules onto HOPG is mainly driven by hydrophobic interactions [15], being strongly influenced by the hydrophobicity of the constituent nucleotides, the ODN molecular mass and the formation of secondary structures. As expected, the eTBA presents an increased adsorption onto HOPG when compared with TBA, due to an enhanced hydrophobic character given by the larger number of guanine bases, especially at the molecule extremities.

Additionally, G-rich ODN sequences that contain sections of guanines can form G-quadruplex structures comprised of stacked tetrads, each one of the tetrads formed by a planar association of four guanines by Hoogsteen hydrogen bonding. Therefore, the guanine bases that enter in the TBA and eTBA composition influence the ODN hydrophobicity directly, through the intrinsic hydrophobic character of the aromatic ring, and indirectly, by allowing the ODN sequences to establish quadruplex conformations. In this context, the interaction of the single-stranded molecules with HOPG is facilitated because they have the bases exposed to the solution and free to undergo hydrophobic interactions, when compared to the ODNs with quadruplex morphology that have the bases protected by the sugarphosphate backbones. Indeed, the AFM images of the TBA and eTBA modified HOPG surfaces show the formation of $1.5-1.9 \mathrm{~nm}$ height spherical and rod-like shape aggregates due to the adsorption of ODNs presenting G-quartet configurations, as also observed for telomeric G-rich sequences on mica [16]. The adsorption of single-stranded molecules led to the formation of polymeric structures and films of approximately $0.8 \mathrm{~nm}$ height.

The stabilisation of the quadruplex structures requires the presence of metal ions, in particular alkali metals, and the order of preference is $\mathrm{K}^{+}>\mathrm{Na}^{+}$. The ions are positioned in the interior channel that is formed at the centre of each G-quartet, coordinating the $\mathrm{O} 6$ atoms of the guanines. The AFM results previously described were obtained in the presence of $\mathrm{Na}^{+}$ions ( $\mathrm{pH} 7.00 .1 \mathrm{M}$ phosphate buffer solutions). In order to establish how the presence of $\mathrm{K}^{+}$ ions influences the formation and stabilisation of TBA and eTBA quadruplexes and therefore modifies their pattern of adsorption onto HOPG, the morphological characteristics of the HOPG modified by spontaneous adsorption from $1 \mu \mathrm{g} \mathrm{mL}{ }^{-1}$ TBA or eTBA in $\mathrm{pH}$ 7.0 $0.1 \mathrm{M}$ phosphate buffer after incubation with $100 \mathrm{mM}$ and $200 \mathrm{mM} \mathrm{KCl}$ during different periods of time, were also analysed.

AFM images of the TBA modified HOPG obtained after incubation of TBA with $100 \mathrm{mM} \mathrm{K}^{+}$during 1 hour showed tilted polymeric structures of $0.8 \pm 0.2 \mathrm{~nm}$ height, Figure 1(c). A dramatically reduced adsorption was observed in the presence of $\mathrm{K}^{+}$, when compared with the adsorption of TBA from solutions containing only $\mathrm{Na}^{+}$cations, 


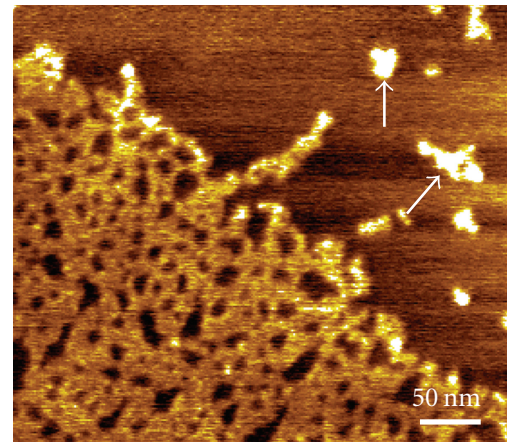

(a)

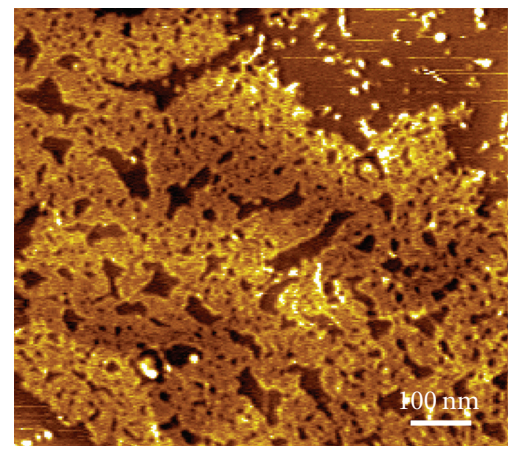

(c)

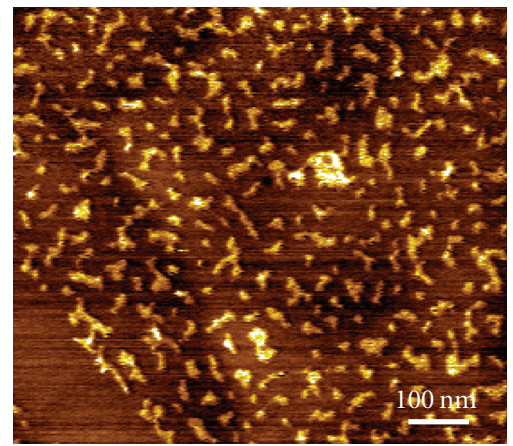

(e)

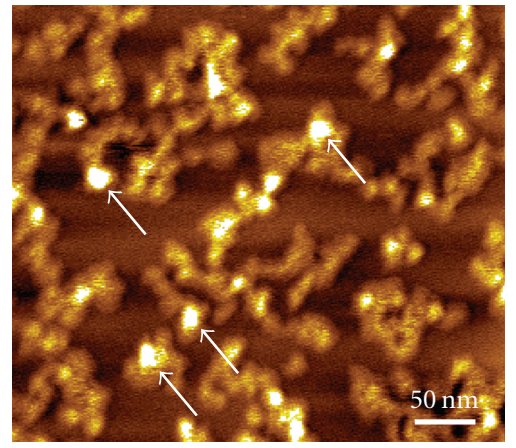

(b)

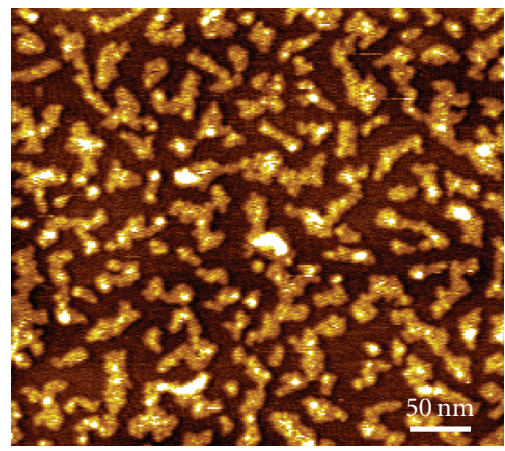

(d)

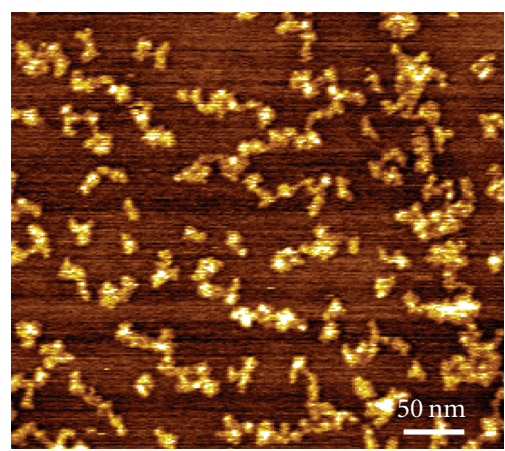

(f)

FIGURE 2: AFM topographical images in air of eTBA molecules, immobilized onto HOPG by spontaneous adsorption during 3 minutes, from solutions of $1 \mu \mathrm{g} \mathrm{mL} \mathrm{g}^{-1}$ eTBA in $\mathrm{pH} 7.00 .1 \mathrm{M}$ phosphate buffer: (a, b) in the absence and (c-f) in the presence of (c, d) $100 \mathrm{mM}$ and (e, f) $200 \mathrm{mM} \mathrm{K}^{+}$, after (c, e) 1 hour and (d, f) 24 hours of incubation.

Figures 1(a) and 1(b). The adsorption decreased further after 24 hours incubation with $\mathrm{K}^{+}$and the adsorbed TBA fragments presented heights of $0.9 \pm 0.3 \mathrm{~nm}$, Figure 1 (d).

AFM images of the eTBA modified HOPG obtained from solutions of eTBA incubated with $100 \mathrm{mM} \mathrm{K}^{+}$during 1 hour, Figure 2(c), showed $0.8 \pm 0.1 \mathrm{~nm}$ height network films and small spherical and rod-like shape aggregates, similar to what was obtained with eTBA in the presence of only $\mathrm{Na}^{+}$, Figures 2(a) and 2(b). After 24 hours incubation in the presence of $\mathrm{K}^{+}$the HOPG coverage decreased significantly, Figure 2(d), although the observed $0.8 \pm 0.1 \mathrm{~nm}$ eTBA lattice still presented small embedded $1.5 \mathrm{~nm}$ height aggregates, corresponding to molecules in G-quartet configurations. AFM images of the eTBA modified HOPG obtained after incubation with $200 \mathrm{mM} \mathrm{K}^{+}$during 1 hour, Figure 2(e), and 24 hours, Figure 2(f), showed, as expected, an even more reduced adsorption, and the fragments observed in the images presented heights of $0.8 \pm 0.1 \mathrm{~nm}$, corresponding only to the adsorption of single-stranded molecules.

AFM images demonstrated that, after the interaction with $\mathrm{K}^{+}$ions during long incubation times, the adsorption pattern of both TBA and eTBA molecules corresponded only to the spontaneous adsorption of a small number of single-stranded molecules present in solution, while no quadruplex TBA/eTBA was observed. This is due to the fact that, in the presence of $\mathrm{K}^{+}$cations, TBA and eTBA form very stable and rigid intramolecular quadruplex configurations, which prevent the interaction of the hydrophobic bases with 


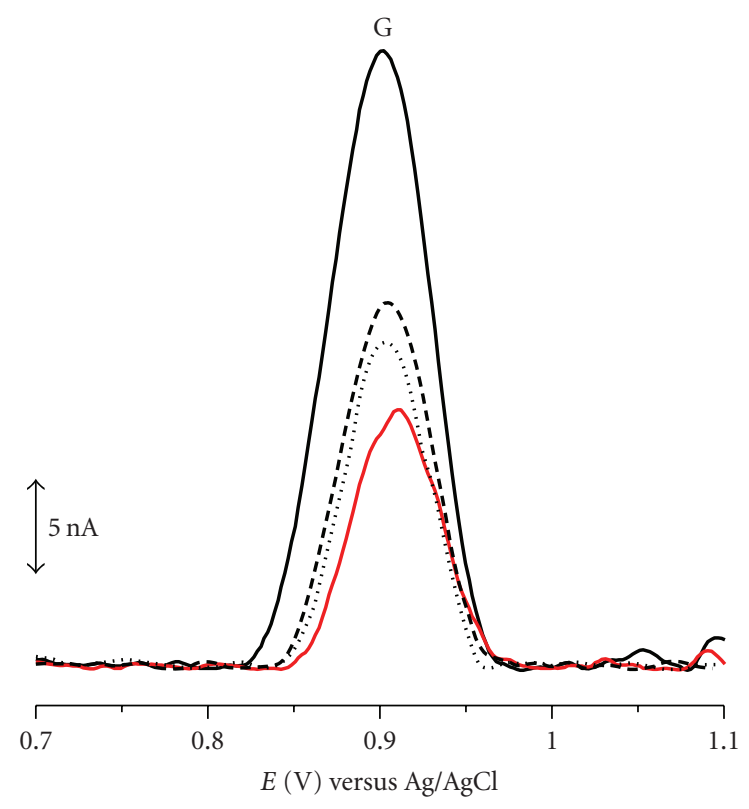

(a)

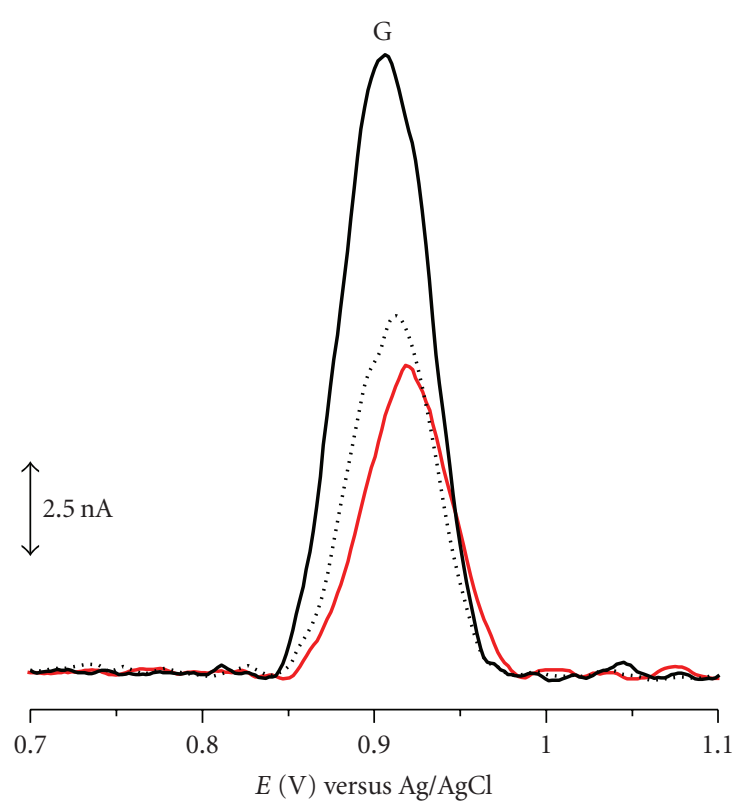

(b)

FIgURE 3: Baseline corrected DPV obtained with the GC electrode in a solution of $1 \mu \mathrm{g} \mathrm{mL}-1 \mathrm{TBA}$ in $\mathrm{pH} 7.00 .1 \mathrm{M}$ phosphate buffer: (a) (solid line) in the absence and in the presence of $100 \mathrm{mM} \mathrm{KCl}$ after (dashed line) 0 hour, (dotted line) 1 hour and (red line) 24 hours of incubation and (b) in the presence of (solid line) 50, (dotted line) 100 and (red line) $200 \mathrm{mM} \mathrm{KCl}$ after 24 hours of incubation.

the HOPG. On the contrary, the presence of only $\mathrm{Na}^{+}$ cations from the solutions of TBA/eTBA in $\mathrm{pH} 7.00 .1 \mathrm{M}$ phosphate buffer led to the formation of less stable quadruplex TBA/eTBA morphologies that are locally destabilised by the HOPG hydrophobic surface, inducing their consequent adsorption.

3.2. Voltammetric Characterisation of TBA and eTBA. DP voltammograms were recorded in solutions containing $1 \mu \mathrm{g} \mathrm{mL}^{-1}$ TBA in $\mathrm{pH} 7.00 .1 \mathrm{M}$ phosphate buffer before and after incubation with $100 \mathrm{mM} \mathrm{K}^{+}$ions during different periods of time, Figure 3(a). Between measurements, the GC electrode surface was always polished in order to ensure a clean surface to avoid possible problems from the adsorption of TBA. On the voltammograms obtained in the solution containing only TBA, one main anodic peak $G$ occurred at $E_{\mathrm{pa}}=+0.91 \mathrm{~V}$. This peak is due to the oxidation of guanine residues, since the oxidation of thymine occurs at much higher positive potential, near the potential of oxygen evolution and therefore it is more difficult to detect [17]. Upon addition of $\mathrm{K}^{+}$ions, a decrease of the guanine oxidation peak occurred in a time-dependent manner reaching constant currents after 24 hours of incubation, Figure 3(a). This process is related to a decrease of the concentration of free/uncomplexed guanine residues available for oxidation and can be explained considering the TBA quadruplex structure in the presence of $\mathrm{K}^{+}$ions. Upon the formation of G-quartets, the guanine electroactive centers are hidden inside the rigid quadruplex being unable to reach the GC electrode surface and consequently unavailable for electrochemical oxidation. Thus, the occurrence of a smaller guanine oxidation peak with increasing incubation time is attributed to guanine molecules that were not involved in the formation of G-quartets.

A similar experiment was carried out in which $1 \mu \mathrm{g} \mathrm{mL}^{-1}$ TBA in $\mathrm{pH}$ 7.0 0.1 M phosphate buffer was incubated during 24 hours with different concentrations of $\mathrm{K}^{+}$ions. The DP voltammograms recorded in these solutions, Figure 3(b), showed a decrease of the guanine oxidation peak dependent on the $\mathrm{K}^{+}$ions concentration, relative to the guanine oxidation peak obtained in a solution containing only TBA. Thus, by increasing the $\mathrm{K}^{+}$ions concentration, the guanine oxidation peak recorded after 24 hours of incubation gradually decreased and its potential become more positive $(\sim 10 \mathrm{mV})$ in agreement with a greater difficulty for the transition of electrons after the formation of more complex structures.

DP voltammograms were recorded in solutions containing $1 \mu \mathrm{g} \mathrm{mL}^{-1}$ eTBA in $\mathrm{pH}$ 7.0 $0.1 \mathrm{M}$ phosphate buffer before and after incubation with $100 \mathrm{mM} \mathrm{K}^{+}$ions during different periods of time, Figure 4(a). Between measurements, the GC electrode surface was always polished in order to ensure a clean surface to avoid possible problems from the adsorption of eTBA. The voltammograms obtained in the solution containing only eTBA, showed only the guanine oxidation peak G, at $E_{\mathrm{pa}}=+0.91 \mathrm{~V}$. DP voltammograms were also recorded after 0 hour, 1 hour and 24 hours of incubation. It was observed that, upon addition of $\mathrm{K}^{+}$ions, a decrease of guanine oxidation peak occurred in a time-dependent manner reaching constant currents after 24 hours of incubation, Figure 4(a). As in the case of TBA, the occurrence of a smaller guanine oxidation peak with increasing incubation time is attributed to guanine molecules that were not involved in the formation of G-quartets. 


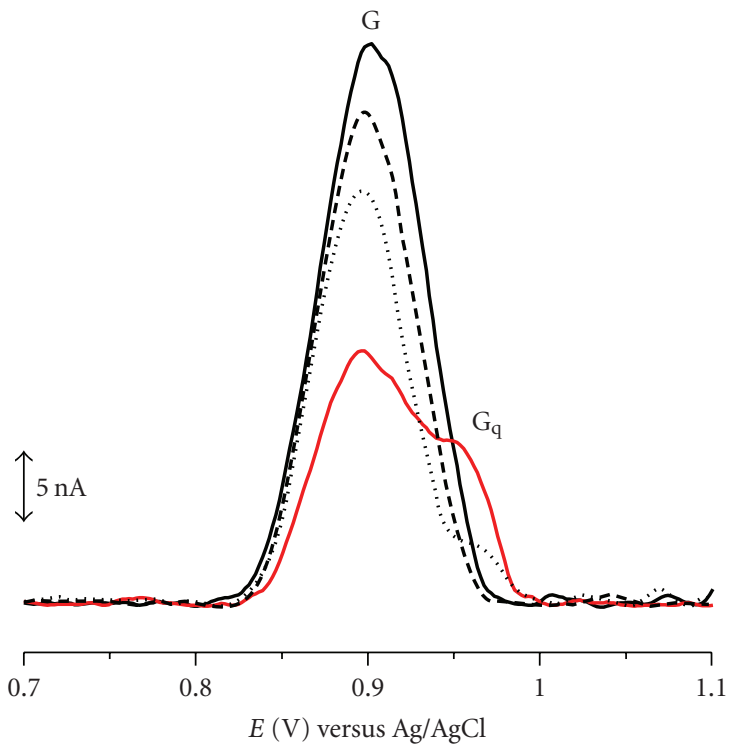

(a)

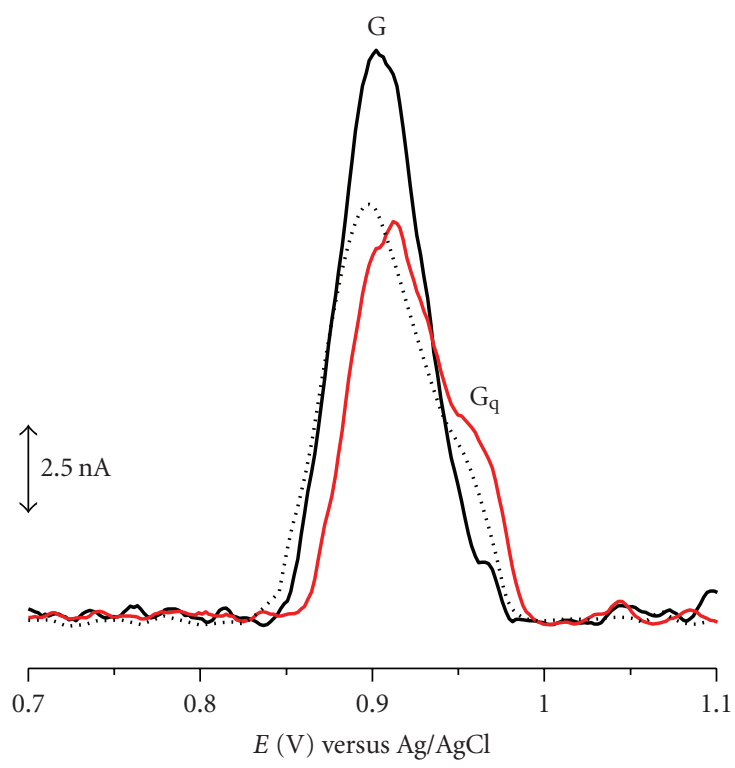

(b)

FIgURE 4: Baseline corrected DPV obtained with the GC electrode in a solution of $1 \mu \mathrm{g} \mathrm{mL} \mathrm{m}^{-1}$ eTBA in pH $7.00 .1 \mathrm{M}$ phosphate buffer: (a) (solid line) in the absence and in the presence of $100 \mathrm{mM} \mathrm{KCl}$ after (dashed line) 0 hour, (dotted line) 1 hour and (red line) 24 hours of incubation and (b) in the presence of (solid line) 50, (dotted line) 100 and (red line) $200 \mathrm{mM} \mathrm{KCl}$ after 24 hours of incubation.

On the other hand, on the voltammograms obtained after 1 hour of incubation of eTBA with $\mathrm{K}^{+}$ions, a new small peak $\mathrm{G}_{\mathrm{q}}$ appeared at $E_{\mathrm{pa}}=+0.97 \mathrm{~V}$ and its current increased with incubation time reaching constant values after 24 hours of incubation, Figure 4(a). This new peak is attributed to the oxidation of G-quartets and its higher potential value relative to guanine itself can be explained considering the greater difficulty for the transition of electrons from the inside of the rigid quadruplex to the GC electrode surface than from the more flexible form of single stranded eTBA where guanine residues can reach the surface leading to higher peak currents [17]. Although this peak did not appear during the experiments with TBA, this can be due to the formation of G-quartets below the detection limit of the technique. On the contrary, in the case of eTBA a higher number of guanine residues are available and thus a higher number of G-quartets are expected, which leads to the occurrence of larger oxidation peaks.

In another experiment, $1 \mu \mathrm{g} \mathrm{mL}^{-1}$ eTBA in $\mathrm{pH} 7.0$ $0.1 \mathrm{M}$ phosphate buffer was incubated during 24 hours with different concentrations of $\mathrm{K}^{+}$ions, Figure 4(b). The DP voltammograms recorded in these conditions showed the decrease of guanine peak current $G$ with increasing $\mathrm{K}^{+}$ concentration up to $200 \mathrm{mM}$ where it reached a constant value. On the other hand, peak $\mathrm{G}_{\mathrm{q}}$ occurred with a small current after incubation with low $\mathrm{K}^{+}$ions and also increased with increasing $\mathrm{K}^{+}$concentration.

\section{Conclusions}

The redox behaviour and adsorption process of two thrombin-binding aptamer sequences were studied at room temperature, using AFM and voltammetry at HOPG and
GC electrodes, in the presence/absence of $\mathrm{Na}^{+}$and $\mathrm{K}^{+}$ cations.

Due to the formation of very stable and rigid intramolecular quadruplex configurations in the presence of $\mathrm{K}^{+}$cations, both TBA and eTBA adsorb less onto HOPG, compared to the adsorption in the presence of only $\mathrm{Na}^{+}$ cations present in the $\mathrm{pH}$ 7.0 $0.1 \mathrm{M}$ phosphate buffer. This is due to the fact that a larger number of intramolecular quadruplexes is formed, which were more stable due to the incorporation of $\mathrm{K}^{+}$, which prevents their hydrophobic interaction with HOPG. On the contrary, the presence of only $\mathrm{Na}^{+}$cations led to the formation of less stable quadruplexes that are locally destabilised by the HOPG hydrophobic surface, inducing their adsorption together with single-stranded sequences.

Voltammetric studies showed that both TBA and eTBA are oxidized at GC electrode and the only electrochemical signal is due to the oxidation of guanine residues. Upon addition of $\mathrm{K}^{+}$ions, both TBA and eTBA folded into G-quadruplex structures and this process was observed by the decreasing of the guanine oxidation peak and the occurrence of a new peak at higher potential values due to the oxidation of G-quartets. The difference in the oxidation potential of guanine and G-quartets is attributed to the difficulty of the transition of electrons from the inside of the rigid quadruplex to the GC electrode surface than from the more flexible form of single-stranded ODNs whose guanine residues can reach the surface leading to higher peak currents.

\section{Acknowledgments}

Financial support from Fundação para a Ciência e Tecnologia (FCT), Post-Doctoral Grant SFRH/BPD/36110/2007 (V. C. 
Diculescu), projects PTDC/QUI/65255/2006 and PTDC/ QUI/098562/2008, POCI 2010 (cofinanced by the European Community Fund FEDER), CEMUC-R (Research Unit 285), and COST Action MP0802 (Self-assembled guanosine structures for molecular electronic devices (G4-net)) is gratefully acknowledged.

\section{References}

[1] V. Dapić, V. Abdomerović, R. Marrington, et al., "Biophysical and biological properties of quadruplex oligodeoxyribonucleotides," Nucleic Acids Research, vol. 31, no. 8, pp. 20972107, 2003.

[2] L. Oganesian and T. M. Bryan, "Physiological relevance of telomeric G-quadruplex formation: a potential drug target," BioEssays, vol. 29, no. 2, pp. 155-165, 2007.

[3] S. Cogoi and L. E. Xodo, "G-quadruplex formation within the promoter of the KRAS proto-oncogene and its effect on transcription," Nucleic Acids Research, vol. 34, no. 9, pp. 2536 2549, 2006.

[4] R. F. Macaya, P. Schultze, F. W. Smith, J. A. Roe, and J. Feigon, "Thrombin-binding DNA aptamer forms a unimolecular quadruplex structure in solution," Proceedings of the National Academy of Sciences of the United States of America, vol. 90, no. 8, pp. 3745-3749, 1993.

[5] A. D. Ellington and J. W. Szostak, "In vitro selection of RNA molecules that bind specific ligands," Nature, vol. 346, no. 6287, pp. 818-822, 1990.

[6] M. K. Beissenhirtz and I. Willner, "DNA-based machines," Organic and Biomolecular Chemistry, vol. 4, no. 18, pp. 33923401, 2006.

[7] M. del Toro, R. Gargallo, R. Eritja, and J. Jaumot, "Study of the interaction between the G-quadruplex-forming thrombinbinding aptamer and the porphyrin 5,10,15,20-tetrakis-(Nmethyl-4-pyridyl)-21,23H-porphyrin tetratosylate," Analytical Biochemistry, vol. 379, no. 1, pp. 8-15, 2008.

[8] Z. Tang, D. Shangguan, K. Wang, et al., "Selection of aptamers for molecular recognition and characterization of cancer cells," Analytical Chemistry, vol. 79, no. 13, pp. 4900-4907, 2007.

[9] B. Chakraborty, Z. Jiang, Y. Li, and H.-Z. Yu, "Rational design and performance testing of aptamer-based electrochemical biosensors for adenosine," Journal of Electroanalytical Chemistry, vol. 635, no. 2, pp. 75-82, 2009.

[10] E. Torres-Chavolla and E. C. Alocilja, "Aptasensors for detection of microbial and viral pathogens," Biosensors and Bioelectronics, vol. 24, no. 11, pp. 3175-3182, 2009.

[11] A. Numnuam, K. Y. Chumbimuni-Torres, Y. Xiang, et al., "Aptamer-based potentiometric measurements of proteins using ion-selective microelectrodes," Analytical Chemistry, vol. 80, no. 3, pp. 707-712, 2008.

[12] C. Wang, M. Zhang, G. Yang, et al., "Single-stranded DNA aptamers that bind differentiated but not parental cells: subtractive systematic evolution of ligands by exponential enrichment," Journal of Biotechnology, vol. 102, no. 1, pp. 1522, 2003.

[13] K. N. Morris, K. B. Jensen, C. M. Julin, M. Weil, and L. Gold, "High affinity ligands from in vitro selection: complex targets," Proceedings of the National Academy of Sciences of the United States of America, vol. 95, no. 6, pp. 2902-2907, 1998.

[14] J. A. Kelly, J. Feigon, and T. O. Yeates, "Reconciliation of the X-ray and NMR structures of the thrombin-binding aptamer d(GGTTGGTGTGGTTGG)," Journal of Molecular Biology, vol. 256, no. 3, pp. 417-422, 1996.
[15] A.-M. Chiorcea Paquim, T. S. Oretskaya, and A. M. Oliveira Brett, "Adsorption of synthetic homo- and heterooligodeoxynucleotides onto highly oriented pyrolytic graphite: atomic force microscopy characterization," Biophysical Chemistry, vol. 121, no. 2, pp. 131-141, 2006.

[16] T. C. Marsh, J. Vesenka, and E. Henderson, "A new DNA nanostructure, the G-wire, imaged by scanning probe microscopy," Nucleic Acids Research, vol. 23, no. 4, pp. 696700, 1995.

[17] A. M. Oliveira Brett, V. C. Diculescu, A. M. Chiorcea-Paquim, and S. H. P. Serrano, "Chapter 20 DNA-electrochemical biosensors for investigating DNA damage," Comprehensive Analytical Chemistry, vol. 49, pp. 413-437, 2007. 

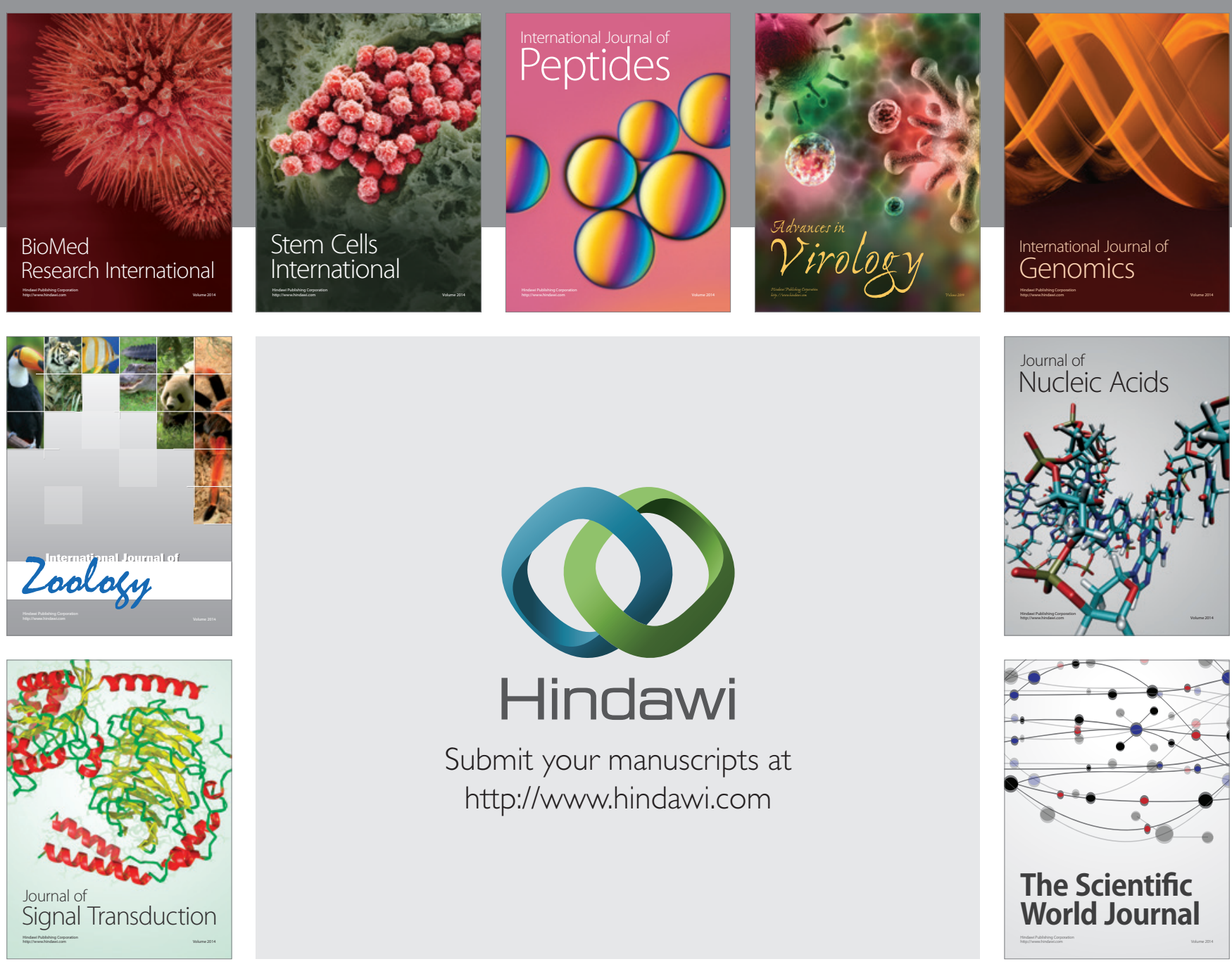

Submit your manuscripts at

http://www.hindawi.com
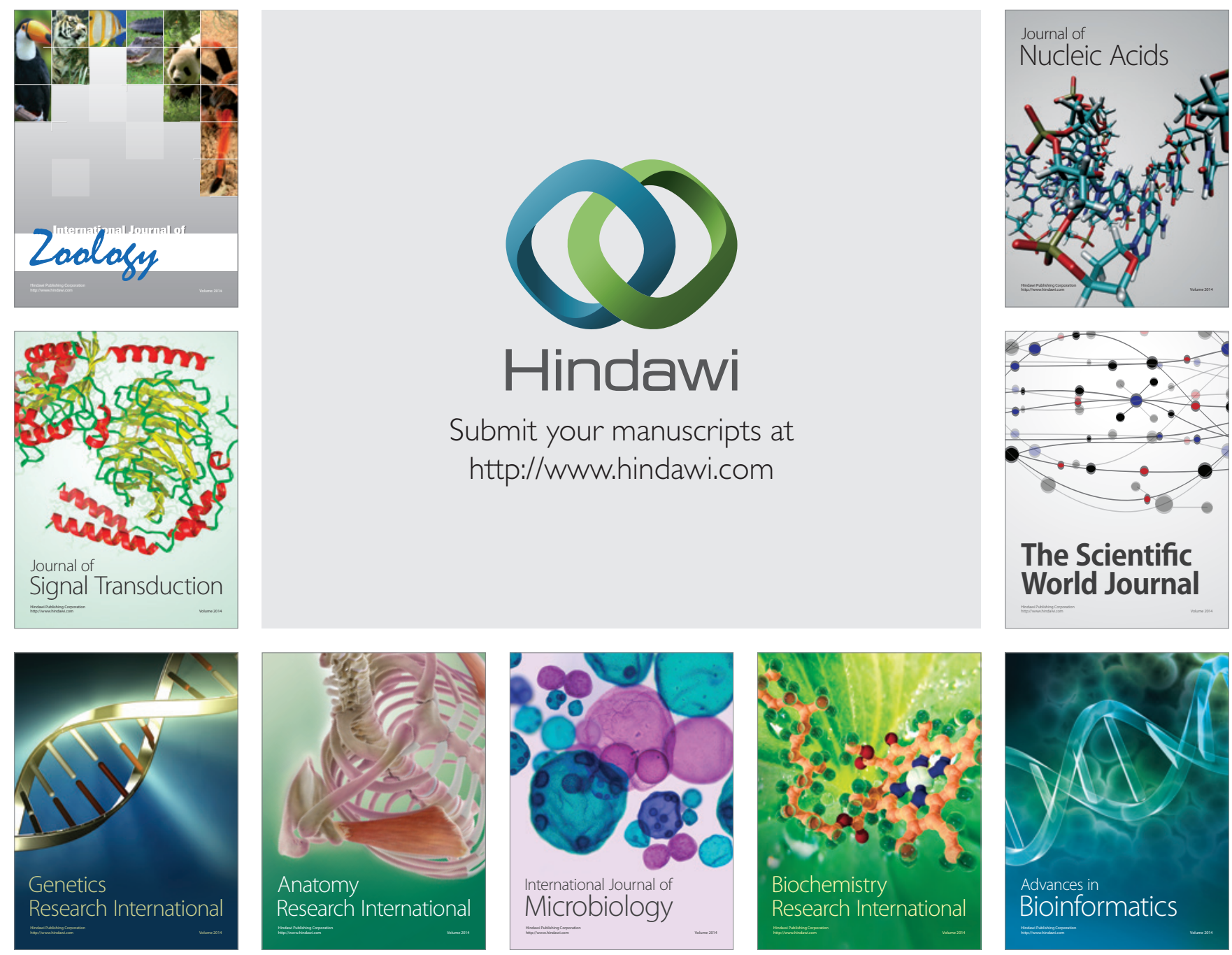

The Scientific World Journal
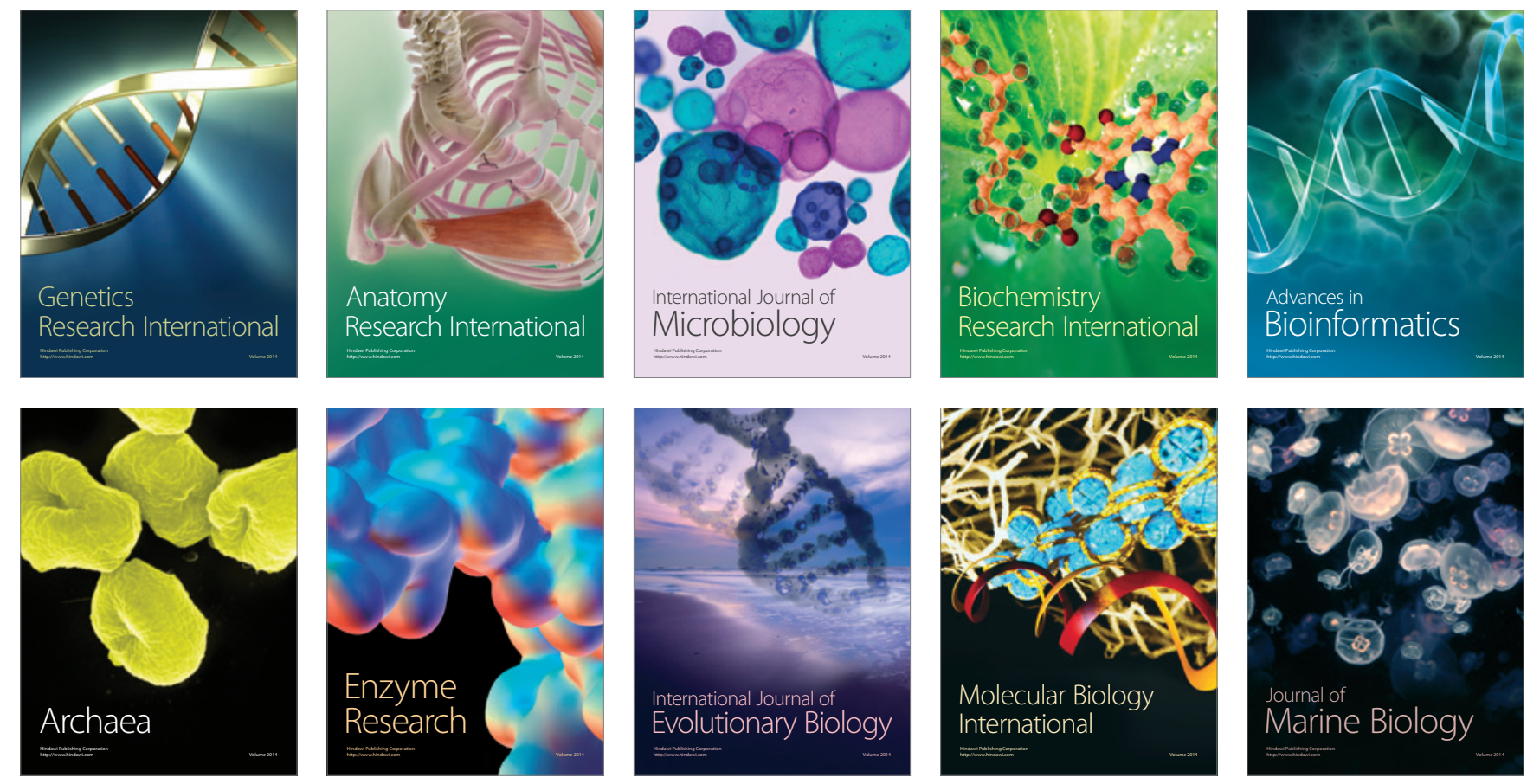